\title{
Comprehensive transcriptomic and proteomic analyses of antroquinonol biosynthetic genes and enzymes in Antrodia camphorata
}

\author{
Xiaofeng Liu, Yongjun Xia, Yao Zhang, Caiyun Yang, Zhiqiang Xiong, Xin Song and Lianzhong Ai* (i)
}

\begin{abstract}
Antroquinonol (AQ) has several remarkable bioactivities in acute myeloid leukaemia and pancreatic cancer, but difficulties in the mass production of AQ hamper its applications. Currently, molecular biotechnology methods, such as gene overexpression, have been widely used to increase the production of metabolites. However, AQ biosynthetic genes and enzymes are poorly understood. In this study, an integrated study coupling RNA-Seq and isobaric tags for relative and absolute quantitation (iTRAQ) were used to identify $A Q$ synthesis-related genes and enzymes in Antrodia camphorata during coenzyme $\mathrm{Q}_{0}$-induced fermentation (FM). The upregulated genes related to acetyl-CoA synthesis indicated that acetyl-CoA enters the mevalonate pathway to form the farnesyl tail precursor of AQ. The metE gene for an enzyme with methyl transfer activity provided sufficient methyl groups for AQ structure formation. The CoQ2 and ubiA genes encode $p$-hydroxybenzoate polyprenyl transferase, linking coenzyme $Q_{0}$ and the polyisoprene side chain to form coenzyme $\mathrm{Q}_{3}$. NADH is transformed into NAD+ and releases two electrons, which may be beneficial for the conversion of coenzyme $Q_{3}$ to $A Q$. Understanding the biosynthetic genes and enzymes of $A Q$ is important for improving its production by genetic means in the future.
\end{abstract}

Keywords: Antrodia camphorata, Antroquinonol, Transcriptome, iTRAQ, Q-PCR

\section{Key points}

1. RNA-Seq and iTRAQ were used to identify AQ synthesis-related genes and enzymes.

2. Ubiquinone and other terpenoid-quinone biosynthesis pathway was upregulated.

3. Genes such as metE, $C o Q 2$, and $u b i A$ are important for AQ production.
*Correspondence: ailianzhong1@126.com

Shanghai Engineering Research Center of Food Microbiology, School of Medical Instrument and Food Engineering, University of Shanghai for Science and Technology, 516 Jungong Road, Shanghai 200093, People's Republic of China

\section{Introduction}

Antrodia camphorata, a unique basidiomycete, is indigenous species of Taiwan. It has been used as a traditional medicine for treat diverse discomforts such as abdominal pain, hangover, and diarrhea (Wu et al. 1997). The fruiting body of $A$. camphorata is rich in bioactive metabolites, but the fruiting body can only grow on the rare Cinnamomum kanehirae Hayata at an extremely slow rate. The mycelium of $A$. camphorata can easily be cultured on a large scale via submerged fermentation, but it has been found to be deficient in specifically bioactive metabolites, such as antroquinonol (AQ) (Lu et al. 2013). AQ, an ubiquinone derivative, was identified from the solid-state fermented mycelium of $A$. camphorata in 2007 (Lee et al. 2007). The remarkably therapeutic activities of AQ in many diseases have been documented, 
including liver and kidney diseases (Angamuthu et al. 2019), Alzheimer's disease (Chang et al. 2015), and cancer (Chiang et al. 2010).

In a previous study, it is successful to stimulate the biosynthesis of $A Q$ in submerged fermentation by addition of precursor coenzyme $\mathrm{Q}_{0}$ (Xia et al. 2018). Therefore, we hypothesize that the addition of coenzyme $\mathrm{Q}_{0}$ would stimulate the expression of genes involved in AQ synthesis. However, a lack research on the molecular genetics of related metabolites in A. camphorata, such as transcriptomic and proteomic resources, currently hinders such studies on AQ biosynthetic genes and enzymes. Largescale sequencing techniques have been widely used, which improve the efficiency of understanding the differential gene expression patterns within microorganism (MacLean et al. 2009; Juan et al. 2010).

In the present study, we focused on the gene transcriptional and protein expression features of A. camphorata S-29 in submerged fermentation with and without the precursor coenzyme $\mathrm{Q}_{0}$ using second-generation sequencing on the Illumina HiSeq ${ }^{\mathrm{TM}} 2000$ platform. We further validated functional genes associated with AQ synthesis through quantitative polymerase chain reaction (q-PCR). Two "omics" levels of analyses indicated that the formation of AQ constitutes a highly complicated and genetically programmed process that requires the participation of multiple regulators. This investigation advances our understanding of genes and enzymes involved in AQ synthesis during FM.

\section{Materials and methods}

\section{Microorganism and cultivation}

The microorganism used in this study was $A$. camphorata S-29, which was deposited in the China General Microbiological Culture Collection Center(CGMCC No. 9590). Seed medium was perpared according to the method of Xia et al. (2018).

The fermentation medium was prepared according to our previous report (Liu et al. 2020). For the investigation on the regulatory mechanisms, $0.3 \mathrm{~g} / \mathrm{L}$ coenzyme $\mathrm{Q}_{0}$ was filtered and added into the fermentation broth. The experiments were carried out with three biological repeats. Mycelia in the fermentation broth were collected by centrifugation at $3000 \mathrm{~g}$ for $10 \mathrm{~min}$ at $4{ }^{\circ} \mathrm{C}$. The mycelia sediment was washed twice with $40 \mathrm{~mL}$ of potassium phosphate buffer, rapidly frozen in liquid nitrogen, and stored at $-80^{\circ} \mathrm{C}$ for further use.

The absence of precursor (coenzyme $\mathrm{Q}_{0}$ ) during fermentation was regarded as conventional submerged fermentation (KB). Adding coenzyme $\mathrm{Q}_{0}$ during fermentation was named as coenzyme $\mathrm{Q}_{0}$-induced fermentation (FM). KB4 (or FM4), KB5 (or FM5), and KB10 (or FM10) representing the mycelia of $A$. camphorata S-29 were collected on days 4,5 , and 10 , respectively.

\section{Differential transcriptome analysis}

The days 4, 5, and 10 samples were sequenced by Shanghai Majorbio Biopharm Technology Co., Ltd (Shanghai, China), with the Illumina HiSeq ${ }^{\mathrm{TM}} 2000$ platform (Bai et al. 2015). The raw data were filtered by removing the low quality reads, sequences of length less than $20 \mathrm{bp}$, and reads containing adapters to obtain clean data. A rapid comparison of the sequencing data with a reference genome was performed by Tophat 2 software, which can also be used to detect events such as variable shear and gene fusion (Trapnell et al. 2012). Analyses of sequence saturation, gene coverage, and duplicate reads in the transcriptome were performed using RSeQC-2.6.3 software (Vera Alvarez et al. 2019). Analysis of gene expression was performed using RSEM software (Li and Dewey 2011). Furthermore, edgeR software was used to calculate the differential expression based on gene read count data (Robinson et al. 2010). The differentially expressed genes (DEGs) were classified by the Gene Ontology (GO, http:// geneontology.org/) database according to the biological process, cellular components, and molecular functions. KOBAS software (Peking University, Beijing, China) was used to test the enrichment of the DEGs; in particular, the Kyoto Encyclopedia of Genes and Genomes (KEGG, http://www.genome.jp/kegg/) pathways (Xie et al. 2011).

\section{Protein quantification by isobaric tags for relative and absolute quantitation (iTRAQ)}

Protein quantification by iTRAQ was carried out according to the method of Lu et al. (2017). The mixed peptides were sequenced by Majorbio Biopharm Technology Co., Ltd (Shanghai, China). All identified proteins were annotated by GO and KEGG. Differential proteins were screened based on differential multiples and $P$-values. The data were analysed by the Majorbio I-Sanger Cloud Platform (https://cloud.majorbio.com/).

The enrichment ratio (ER) was calculated as follow: $\mathrm{ER}=\mathrm{CN} / \mathrm{BN}$, where $\mathrm{CN}$ is the number of proteins (or genes), which were enriched to this KEGG in the protein (or gene) set, and $\mathrm{BN}$ is the number of proteins (or genes), which were enriched to this KEGG among all annotated proteins (or genes).

\section{Validation of $A Q$ synthesis-related genes using q-PCR}

Reverse transcription of cDNA was performed according to the manufacturer's instructions (PrimeScript 1st strand cDNA synthesis kit, Takara, Japan). Q-PCR was performed using LightCycler ${ }^{\circledR} 96$ system (Roche, Switzerland) detection. The $18 S$ rRNA gene of $A$. camphorata S-29 was used as the internal standard. Primers used in 
this research for q-PCR (Additional file 1: Table S1) were designed by Oligo 7.0 software. Relative expression level of gene was quantified based on the $2^{-\Delta \Delta C t}$ method. It was carried out with three biological repeats. Values are given as the means \pm standard deviations $(n=3)$.

\section{Sequencing data}

The FASTQ format raw reads were deposited to the National Center for Biotechnology Information Short Read Archive (NCBI SRA) database (accession: PRJNA543624 and PRJNA622907). The mass spectrometry proteomics data have been deposited to the ProteomeXchange Consortium (http://proteomecentral .proteomexchange.org) via the iProX partner repository with the dataset identifier PXD018004 (Ma et al. 2019).

\section{Results}

\section{Identification of DEGs via transcriptome sequencing}

The highest number of the expressed genes of $A$. camphorata S-29 was from FM5 (7478), followed by KB5
(7470), KB4 (7456), FM4 (7436), KB10 (7409), and FM10 (7356), respectively (Additional file 1: Figure S1). KEGG map shows that the highest number of genes were involved in metabolism. Over 200 genes annotated to the KEGG functional categories of translation, carbohydrate metabolism, folding, sorting and degradation, and amino acid metabolism (Additional file 1: Figure S2). Scatter plots and Venn diagram were used to analyse of DEGs between KB and FM (Fig. 1). As shown in Fig. 1a-c, the DEGs between KB and FM increased gradually from 4 to 10 days. The number of DEGs were 51, 151, and 732 on days 4,5 , and 10 , respectively (Fig. 1d). A total of 35 DEGs were only expressed between KB4 and FM4, with 101 DEGs expressed between KB5 and FM5. In comparison, 677 DEGs were only expressed between KB10 and FM10.

Functional classification of genes by GO and KEGG analysis These genes were classified into $20 \mathrm{GO}$ categories, and all GO categories were then assigned into three main

\section{a}

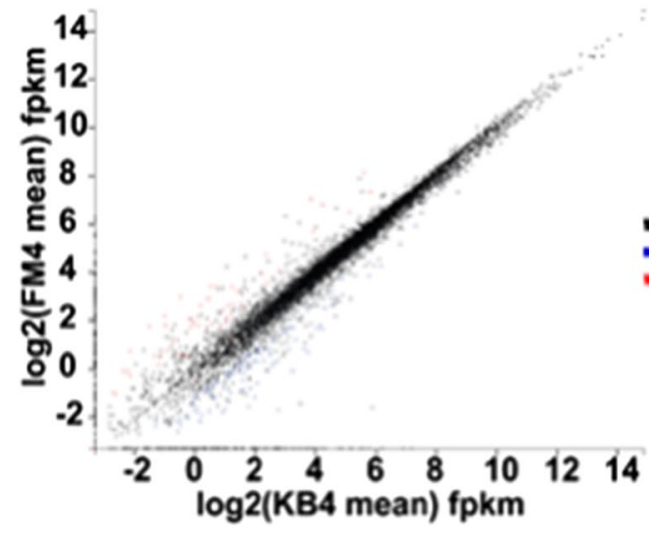

c

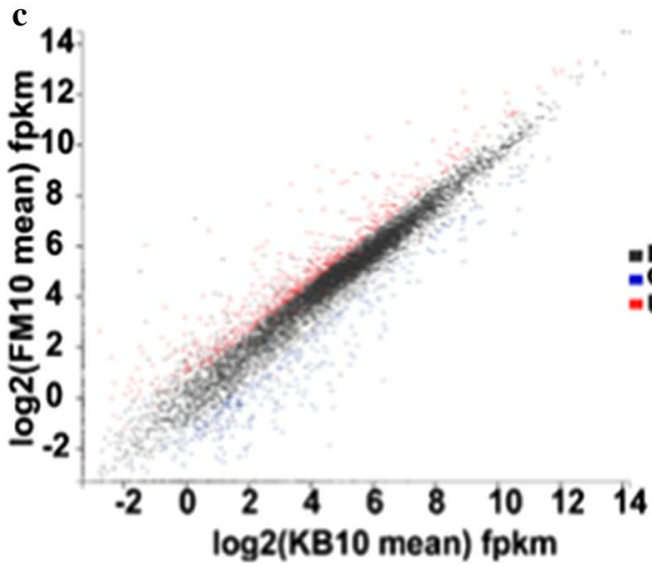

b

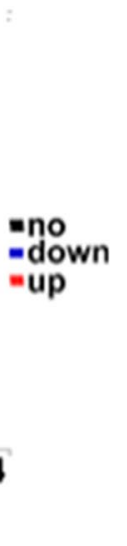

d

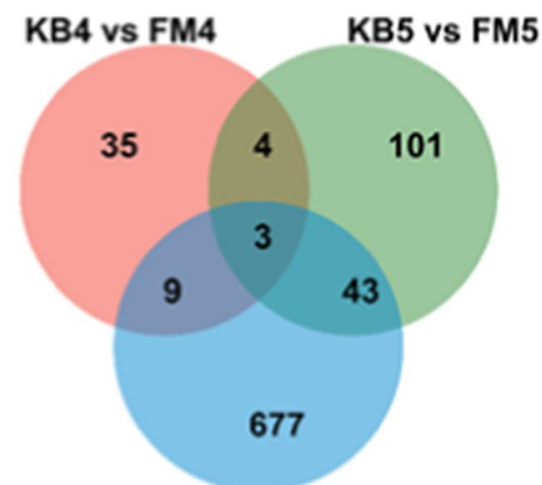

KB10 vs FM10

Fig. 1 DEGs between KB and FM. a-c a scatter plot of the DEGs during FM compared with KB on days 4, 5, and 10, respectively. Significantly upregulated or downregulated genes were labeled with red dots or blue dots, respectively. $\mathbf{d}$ Venn diagram of the DEGs on days 4, 5, and 10, respectively 
categories: biological process, cellular component, and molecular function. As shown in Fig. 2, in the biological processes, 9 significant enrichment items were found. Particularly, a large number of genes involved in metabolic process, single-organism metabolic process, and cellular processes were significantly enriched in FM10 compared to KB10. In the cellular component, 7 significant enrichment items were found. In the molecular functionality, 4 significant enrichment items, especially catalytic activity and binding, were identified in FM10 compared to KB10.

In the early phase of coenzyme $\mathrm{Q}_{0}$ addition (on day 4 ), only the SNARE interactions in vesicular transport pathway was significantly enriched in FM4 compared to KB4 (Additional file 1: Figure S3A, $P<0.05$ ). The arginine and proline metabolism, tryptophan metabolism, and C5-branched dibasic acid metabolism were significantly enriched in FM5 compared to KB5 (Additional file 1: Figure S3B, $P<0.05)$. As shown in Fig. 3, 20 metabolic pathways were significantly enriched on day $10(P<0.05)$. Arginine and proline metabolism, lysine degradation, cyanoamino acid metabolism, citrate cycle (TCA cycle), and valine, leucine and isoleucine degradation pathways were significantly enriched $(P<0.001)$. Amino sugar and nucleotide sugar metabolism pathway had the largest number of enriched the DEGs among 20 most significant enrichment pathways (Table 1). The genes of atoB, $A L D H$, and ECHS1 participated in lysine degradation, valine, leucine and isoleucine degradation, tryptophan metabolism, and fatty acid degradation pathways at the same time. It is noteworthy that the DEGs of ubiquinone and other terpenoid-quinone biosynthesis pathway were CoQ2 and CoQ5 (Table 1). Additional file 1: Table S2 shows that the $\mathrm{CoQ} 2$ gene, which encodes 4-hydroxybenzoate polyprenyltransferase, was upregulated, while the CoQ5 gene, which encodes 2-methoxy-6-polyprenyl1,4-benzoquinol methylase, was downregulated on day 10.

\section{Identification of differentially expressed proteins (DEPs) using ITRAQ}

The iTRAQ is a technique in quantitative proteome that accurately measures large-fold changes in protein expression within dynamic ranges of protein abundance (Juan et al. 2010). Here we used this technique to perform proteomic analyses of the key enzymes involved in AQ biosynthetic pathways. The iTRAQ data show that 44,751 unique peptides, which were assembled into 3987 proteins (Additional file 1: Figure S4). All proteins were then assigned into three main GO categories: biological process (BP), cellular component (CC), and molecular function (MF). As shown in Additional file 1: Figure S5,

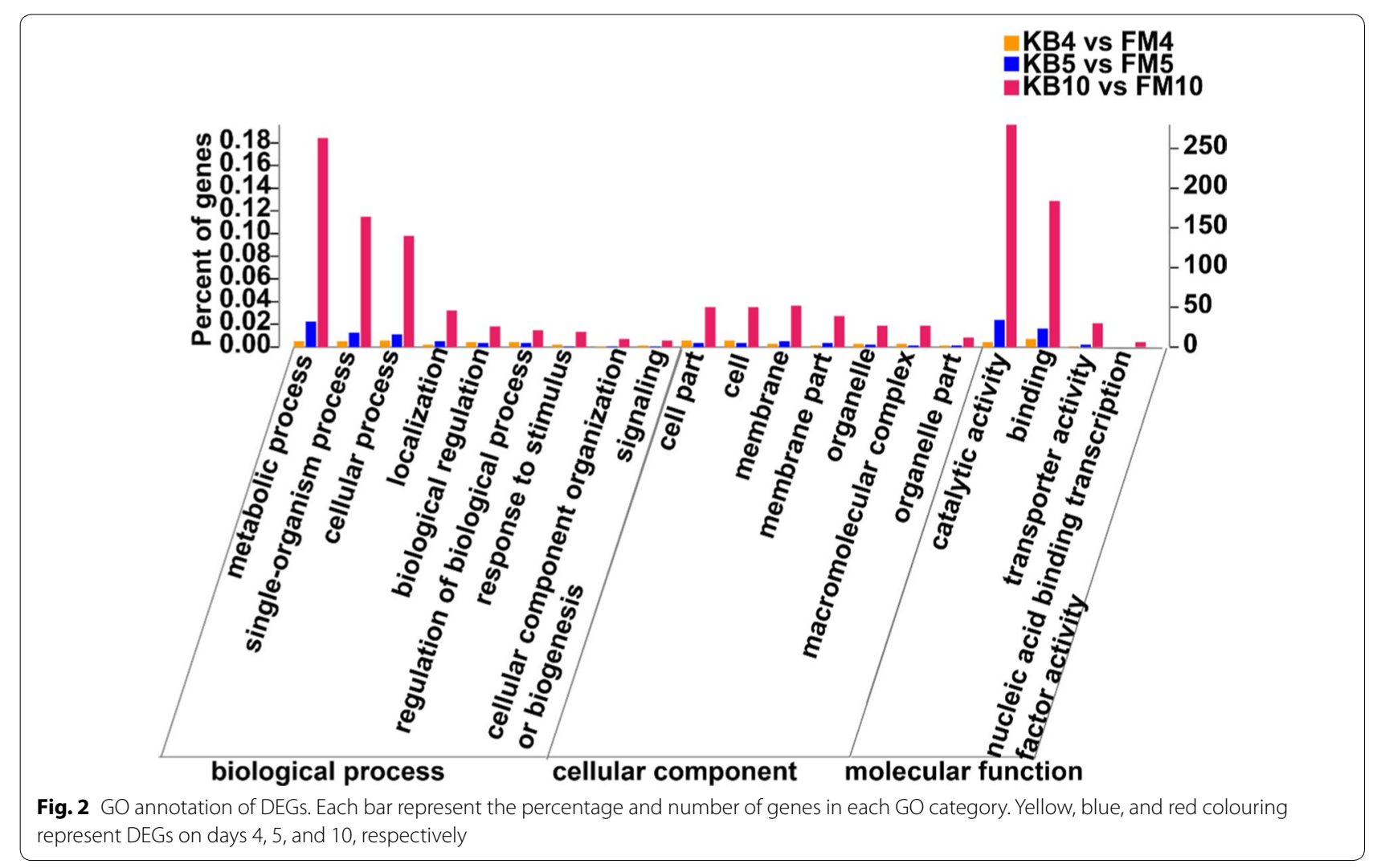



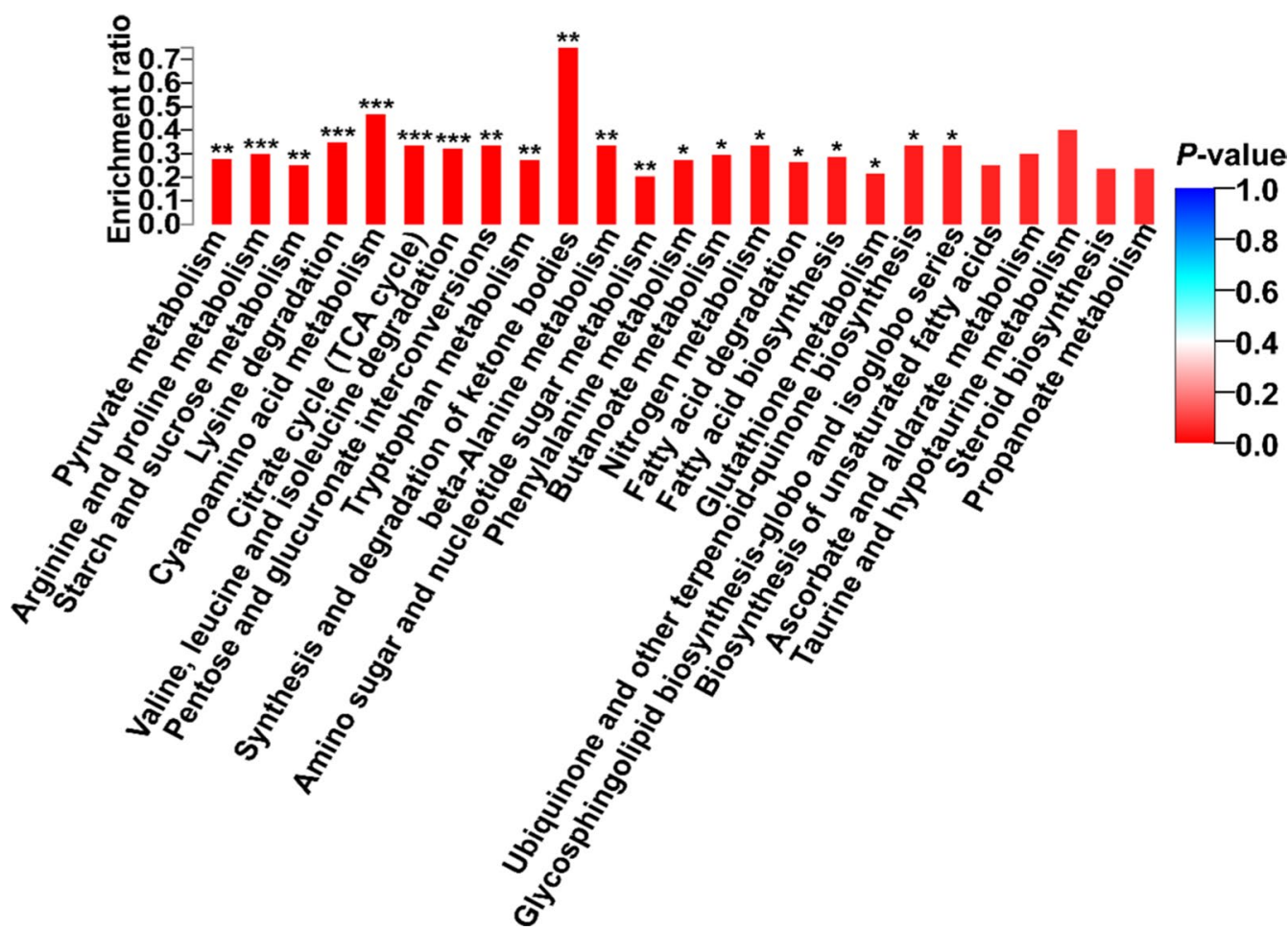

Fig. 3 Analysis of the DEGs between KB10 and FM10 based on the KEGG enrichment map. ${ }^{*} P<0.05,{ }^{* *} P<0.01$, ${ }^{* * *} P<0.001$

Table 1 Annotations of the KEGG pathway for DEGs on day 10

\begin{tabular}{|c|c|c|c|c|}
\hline Pathway & Pathway ID & Ratio in study & $P$-value & Genes \\
\hline Pyruvate metabolism & map00620 & $8 / 190$ & 0.00112 & IIdD; IMS; ACACA; atoB; FH; ALDH; maeB; pckA \\
\hline Arginine and proline metabolism & map00330 & $4 / 190$ & 0.00033 & amiE; PRODH; ALDH; speB \\
\hline Starch and sucrose metabolism & map00500 & $7 / 190$ & 0.00102 & TPS; GPI; E3.2.1.58; UGP2; E2.4.1.34; AGL; bgIX \\
\hline Lysine degradation & map00310 & $6 / 190$ & 0.00069 & SUV39H;OGDH; ALDH; EZH2; ECHS1; atoB \\
\hline Cyanoamino acid metabolism & map00460 & $3 / 190$ & 0.00018 & CD224; ggt; bglX \\
\hline Citrate cycle (TCA cycle) & map00020 & $7 / 190$ & 0.00096 & OGDH; fumC; $A C O ; A C L Y ; S D H A ; p c k A ; C S$ \\
\hline Valine, leucine and isoleucine degradation & map00280 & $7 / 190$ & 0.00062 & E2.3.3.10; atoB; OXCT; IVD; ALDH; ECHS1; HIBADH \\
\hline Pentose and glucuronate interconversions & map00040 & $5 / 190$ & 0.00202 & $X Y L B ; \cup G P 2 ; S O R D ; A L D H ; A R D 1$ \\
\hline Tryptophan metabolism & map00380 & $5 / 190$ & 0.00230 & OGDH; ALDH; ECHS1; amiE; atoB \\
\hline Synthesis and degradation of ketone bodies & map00072 & $3 / 190$ & 0.00295 & $O X C T ; E 2.3 .3 .10 ; a t o B$ \\
\hline Beta-alanine metabolism & map00410 & $3 / 190$ & 0.00427 & $A O C 3 ; A L D H ; E C H S 1$ \\
\hline Amino sugar and nucleotide sugar metabolism & map00520 & $9 / 190$ & 0.00900 & $\begin{array}{l}\text { manC; } H E X A \_B ; \text { GPI; E3.2.1.14; UGP2; E1.6.2.2; } \\
\text { CHS1; nagZ; abfA }\end{array}$ \\
\hline Phenylalanine metabolism & map00360 & $3 / 190$ & 0.01252 & AOC3; PAAH; amiE \\
\hline Butanoate metabolism & map00650 & $5 / 190$ & 0.01611 & OXCT; PAAH; ECHS1;E2.3.3.10; atoB \\
\hline Nitrogen metabolism & map00910 & $2 / 190$ & 0.01972 & NIT-6; NR \\
\hline Fatty acid degradation & map00071 & $3 / 190$ & 0.02598 & $A L D H ; E C H S 1 ; a t o B$ \\
\hline Fatty acid biosynthesis & map00061 & $4 / 190$ & 0.03441 & FAS2; ACACA; fabG; FAS1 \\
\hline Glutathione metabolism & map00480 & $5 / 190$ & 0.03960 & CD224; GST; ggt; PGD; OPLAH \\
\hline Ubiquinone and other terpenoid-quinone biosynthesis & map00130 & $2 / 190$ & 0.04361 & $\mathrm{COQ} 5 ; \mathrm{COQ} 2$ \\
\hline Glycosphingolipid biosynthesis-globo and isoglobo series & map00603 & $2 / 190$ & 0.04361 & galA; HEXA_B \\
\hline
\end{tabular}


a large number of proteins were assigned into the GO categories of biological process and cellular component. Particularly, the proteins of more than 1000 involved in metabolic process, cellular process, single-organism metabolic process, cell, cell part, organelle, catalytic activity, and binding. In total, 3914 proteins did not change significantly, 24 proteins were downregulated, and 49 proteins were upregulated in FM10 compared to KB10 (Additional file 1: Figure S6).

$\mathrm{GO}$ analysis was also used to further elucidate the putative functions of upregulated DEPs on day 10 (Fig. 4). According to the GO analysis, the upregulated DEPs in FM were mainly grouped into the following functions (number of proteins): sulfur amino acid metabolic process (4), one-carbon metabolic process (3), methionine metabolic process (3), sulfur compound biosynthetic process (4), serine family amino acid metabolic process (3), homoserine metabolic process (2), sulfur amino acid biosynthetic process (3), 5-methyltetrahydropteroyltriL-glutamate-dependent methyltransferase activity (2), 5 -methyltetrahydropteroyltriglutamate-homocysteine $S$-methyltransferase activity (2), and $S$-methyltransferase activity $(2)(P<0.001$, Fig. 4 and Additional file 1 : Table S3).
KEGG pathway enrichment analysis was also performed to investigate the enriched pathways of DEPs on day 10 . Seven KEGG pathways were significantly upregulated in FM: cysteine and methionine metabolism $(P<0.001)$, selenocompound metabolism, ubiquinone and other terpenoid-quinone biosynthesis $(P<0.01)$, betalain biosynthesis, glutathione metabolism, glycine, serine and threonine metabolism, and cyanoamino acid metabolism $(P<0.05$, Fig. 5a). One KEGG pathway was significantly downregulated: glycolysis/gluconeogenesis $(P<0.001$, Fig. $5 b)$.

Among these upregulated DEPs in FM, we further identified key enzymes that might be related to the biosynthesis of AQ (Table 2 and Additional file 1: Table S4). Five enzymes (ec:2.5.1.6, ec:2.5.1.18, ec:2.5.1.39, ec:2.5.1.21, and ec:2.5.1.54) are relate to transfer alkyl or aryl groups. Two enzymes (ec:2.1.1.14 and ec:2.1.1.41) are methyltransferases. Seventeen of them are oxidoreductases. The ec:2.1.2.1 and ec:2.1.2.10 are relate to hydroxymethyltransferase and aminomethyltransferase, respectively. Four enzymes (ec:2.3.3.1, ec:2.3.3.8, ec:2.3.3.13, and ec:6.2.1.1) can generate acetyl-CoA during the catalytic reaction.

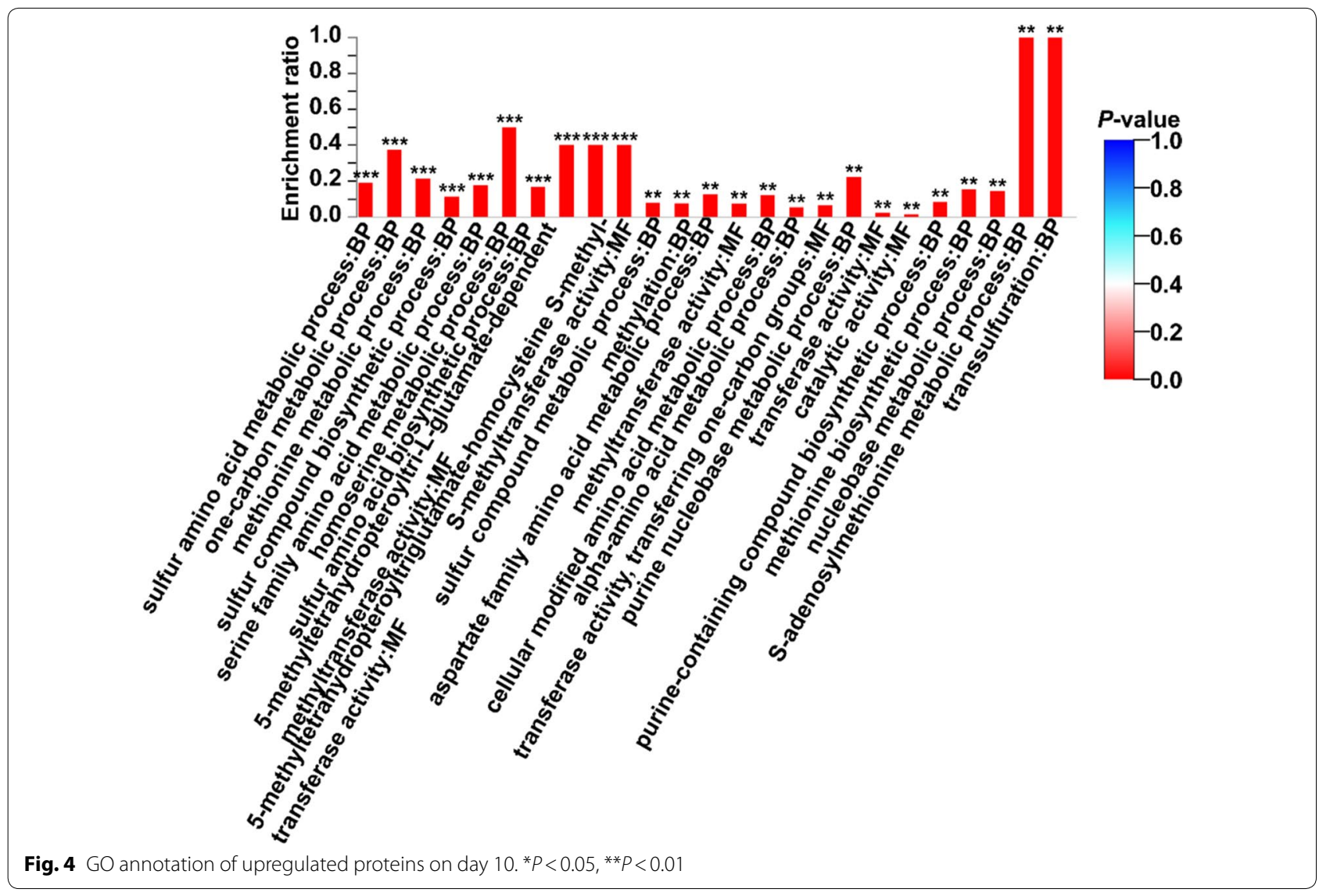




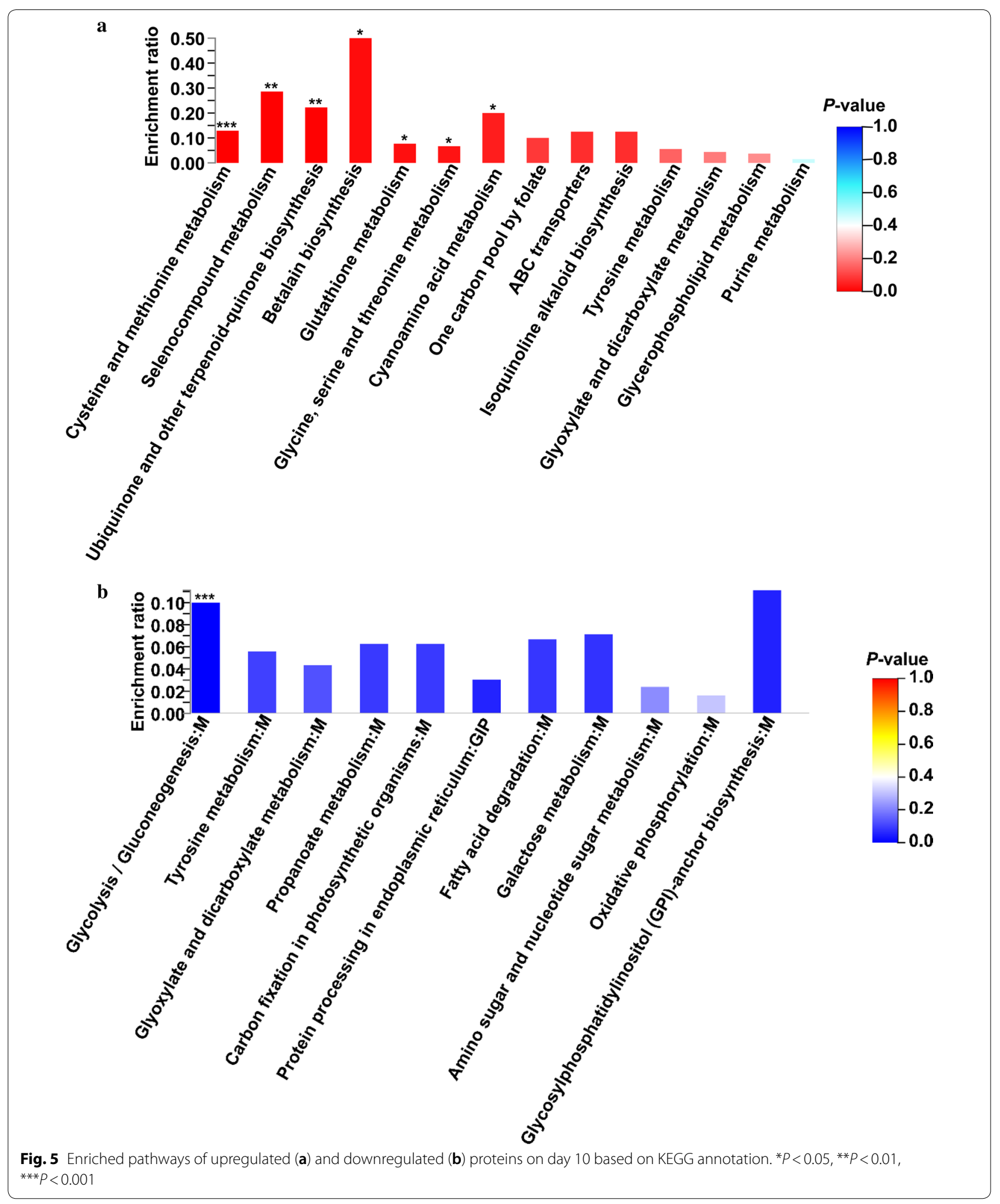


Table 2 Critical upregulated proteins based on proteomic analysis (10-day samples)

\begin{tabular}{|c|c|c|c|c|c|}
\hline Enzyme ID & Gene & $P$-value & Function & KEGG & Class \\
\hline ec:2.5.1.6 & metk & 0.008 & S-Adenosylmethionine synthetase & K00789 & \multirow[t]{5}{*}{ Transferring alkyl or aryl groups, other than methyl groups } \\
\hline ec:2.5.1.18 & GST & $1.5 \times 10^{5}$ & Glutathione S-transferase & K00799 & \\
\hline ec:2.5.1.39 & $\mathrm{CoQ2}$ & 0.038 & 4-Hydroxybenzoate polyprenyltransferase & K06125 & \\
\hline ec:2.5.1.21 & FDFT1 & 0.024 & Farnesyl-diphosphate farnesyltransferase & K00801 & \\
\hline ec:2.5.1.54 & $\operatorname{aroF}$ & 0.010 & 3-Deoxy-7-phosphoheptulonate synthase & K01626 & \\
\hline ec:2.1.1.14 & metE & 0.002 & $\begin{array}{l}\text { 5-Methyltetrahydropteroyltriglutamate- } \\
\text { homocysteine methyltransferase }\end{array}$ & K00549 & \multirow[t]{2}{*}{ Methyltransferases } \\
\hline ec:2.1.1.41 & SMT1 & 0.043 & Sterol 24-C-methyltransferase & K00559 & \\
\hline ec:2.1.2.1 & SHMT & 0.003 & Glycine hydroxymethyltransferase & K00600 & \multirow[t]{2}{*}{ Hydroxymethyl-, formyl- and related transferases } \\
\hline ec:2.1.2.10 & AMT & 0.014 & Aminomethyltransferase & K00605 & \\
\hline ec:2.3.3.1 & CS & 0.015 & Citrate synthase & K01647 & \multirow{3}{*}{$\begin{array}{l}\text { Acyltransferases; Acyl groups converted into alkyl groups on } \\
\text { transfer }\end{array}$} \\
\hline ec:2.3.3.8 & $A C L Y$ & 0.029 & ATP citrate (pro-S)-lyase & K01648 & \\
\hline ec:2.3.3.13 & IMS & 0.029 & 2-Isopropylmalate synthase & K01649 & \\
\hline ec:6.2.1.1 & ACSS1_2 & 0.036 & Acetyl-CoA synthetase & K01895 & Forming carbon-sulfur bonds \\
\hline
\end{tabular}

Integrated analyses of transcriptomic and proteomic datasets of $A$. camphorata S-29

The correlation between the proteome and transcriptome of $A$. camphorata S-29 was demonstrated by scatter plot analysis (Fig. 6). The distribution of the dots with different colors shows that a large number (3589) of mRNAs and proteins did not exhibit changes in expression (black dots, Fig. 6 and Additional file 1: Table S5). On the other hand, a different trend was observed between protein and transcription levels. For instance, 253 genes were upregulated at the transcriptional level but unchanged at the protein level (sky blue). Moreover, 72 genes were downregulated at the transcriptional level but unchanged at the protein level (slate blue). Due to the regulations of

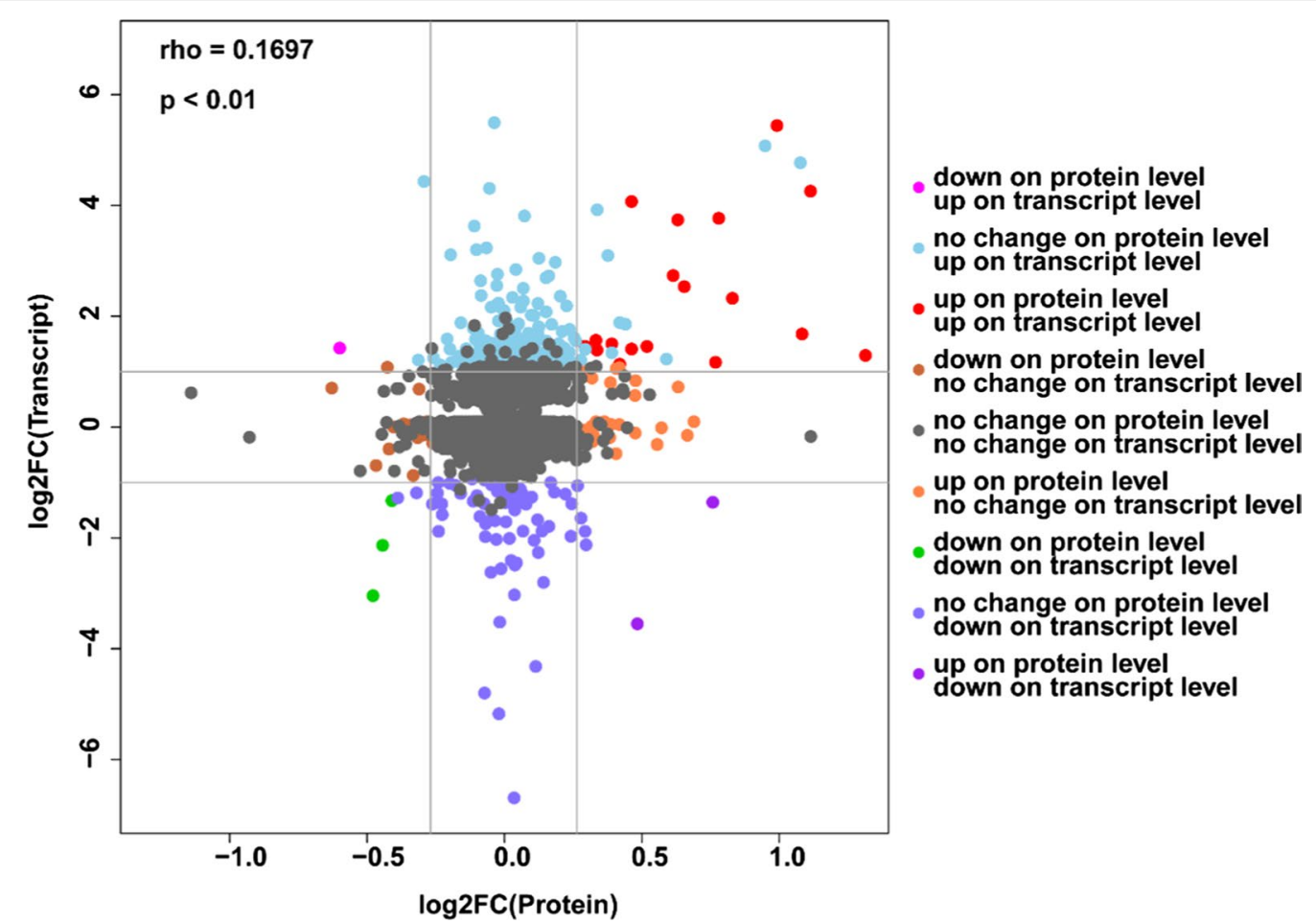

Fig. 6 Correlation analysis of proteomic and transcriptomic data (10-day samples). Differentially expressed mRNAs or proteins are represented by solid dots 
transcription and translation, the mRNA expression levels are not always consistent with protein levels (Washburn et al. 2003). It is noteworthy that 18 unigenes were upregulated at the transcriptional level and the protein level (red).

For the comparative analysis of proteomic and transcriptomic data, we focused on the 9 unigenes that were both upregulated in transcriptomic and proteomic sequencing (Table 3). The $u b i A$ and $C o Q 2$ genes simultaneously participate in the formation of 4-hydroxybenzoate polyprenyltransferase. Of note, among the genes for synthesized benzenoids, PKS63787 gene was the DEG that exhibited the greatest upregulation. Ac-8 cytochrome P450 (CYP) monooxygenase synthesisrelated gene $P 450$ and methyltransferase-related gene $m e t E$ were significantly upregulated in the proteome and transcriptome data. E1.14.13.1, E1.14.13.7, and ADK were associated with the electron transport respiratory chain and energy metabolism.

\section{Validation of gene expression data using q-PCR}

The mRNA expression levels of 5 genes in potential AQ biosynthesis on days $4,5,8$, and 10 were studied (Fig. 7). The mRNA expression levels of 5 genes (ubiA, CoQ2, PKS63787, P450, and metE) in KB and FM were not significantly increased on day 4 (Fig. $7 \mathrm{a}, \mathrm{P}>0.05$ ). Compared with the $\mathrm{KB}$, the expression levels of the $u b i \mathrm{~A}$ and $P 450$ genes increased significantly in FM on day 5 (Fig. $7 \mathrm{~b}, P<0.05$ ). The expression levels of the $u b i A$, CoQ2, PKS63787, P450, and metE genes were significantly higher in FM than in KB at 8 and 10 days (Fig. 7c, $\mathrm{d}, P<0.05)$. Therefore, the gene expression levels with the transcriptome sequencing analyses were consistent with those of the q-PCR analyses (Table 3 and Additional file 1: Table S6). The production of AQ increased significantly on 5-10 days compared with 4 and 5 days
(Additional file 1: Figure S7). The AQ synthesis-related genes expression levels are in line with its production.

\section{Discussion}

AQ is considered one of the most bioactive metabolites in A. camphorata (Lu et al. 2014). The addition of the AQ precursor (coenzyme $\mathrm{Q}_{0}$, orsellinic acid, etc.) via submerged fermentation is considered the most efficient method for the industrial production of AQ. In this study, we used transcriptomic and proteomic data to reveal AQ synthesis-related genes and enzymes during FM. GO enrichment and KEGG pathway analyses of transcriptomic data provided insights into the secondary metabolite biosynthesis pathways. The GO assignment system was used to obtain functional information for the DEGs, further assisting in understanding the distribution of DEG functions at a macro level. Given that fewer differential genes were noted between KB and FM groups in the early stage of adding coenzyme $\mathrm{Q}_{0}$, the number of genes on days 4 and 5 annotated in the GO categories were significantly less than that on day 10 (Figs. 1 and 2). Numerous genes involved in metabolic processes were significantly enriched in FM compared to KB on day 10 . This finding indicates that the addition of the precursor coenzyme $\mathrm{Q}_{0}$ significantly increased the synthesis of metabolites.

KEGG pathway analysis provides classifications that are valuable for studying the complex biological functions of genes. KEGG analysis of transcriptomic data showed that the pathway of fatty acid degradation was significantly enriched in FM (Fig. 3). The ECHS1 gene, which encodes enoyl-CoA hydratase, and the ato $B$ gene, which encodes acetyl-CoA C-acetyltransferase, were upregulated. In fungi, the key metabolic pathway for fatty acid degradation is beta-oxidation. Fatty acids are broken down in a repeating process involving four steps in mitochondria, and each round of processing removes two

Table 3 Significantly upregulated genes and proteins on day 10

\begin{tabular}{lllllll}
\hline Genes & Enzymes & Pro_log2FC & Pro_P-value & Rna_log2FC & Rna_P-value & Function \\
\hline ubiA & ec:2.5.1.39 & 0.63 & 0.038 & 2.15 & $1.59 E-48$ & 4-Hydroxybenzoate polyprenyltransferase \\
CoQ2 & ec:2.5.1.39 & 0.40 & 0.045 & 0.61 & $7.27 E-07$ & 4-Hydroxybenzoate polyprenyltransferase \\
PKS63787 & PKS63787 & 0.44 & 0.036 & 3.51 & $9.49 E-105$ & Synthesize several benzoquinones and benzenoids \\
P450 & P450 monooxygenas & 1.06 & 0.035 & 1.10 & $1.29 E-16$ & ac-8 cytochrome P450 monooxygenas \\
metE & ec:2.1.1.14 & 0.32 & 0.002 & 0.92 & $1.84 E-12$ & 5-Methyltetrahydropteroyltriglutamate-homocyst- \\
& & & & & & eine methyltransferase \\
E1.14.13.1 & ec:1.14.13.1 & 0.76 & 0.027 & 3.47 & $2.73 E-78$ & Salicylate hydroxylase \\
E1.14.13.7 & ec: 1.14 .13 .7 & 0.61 & 0.002 & 2.65 & $2.09 E-68$ & Phenol 2-monooxygenase (NADPH) \\
ADK & ec:2.7.1.20 & 0.27 & 0.014 & 0.75 & $9.61 E-09$ & Adenosine kinase \\
ahcY & ec:3.3.1.1 & 0.37 & 0.010 & 0.98 & $9.87 E-14$ & Adenosylhomocysteinase
\end{tabular}

FC fold change 


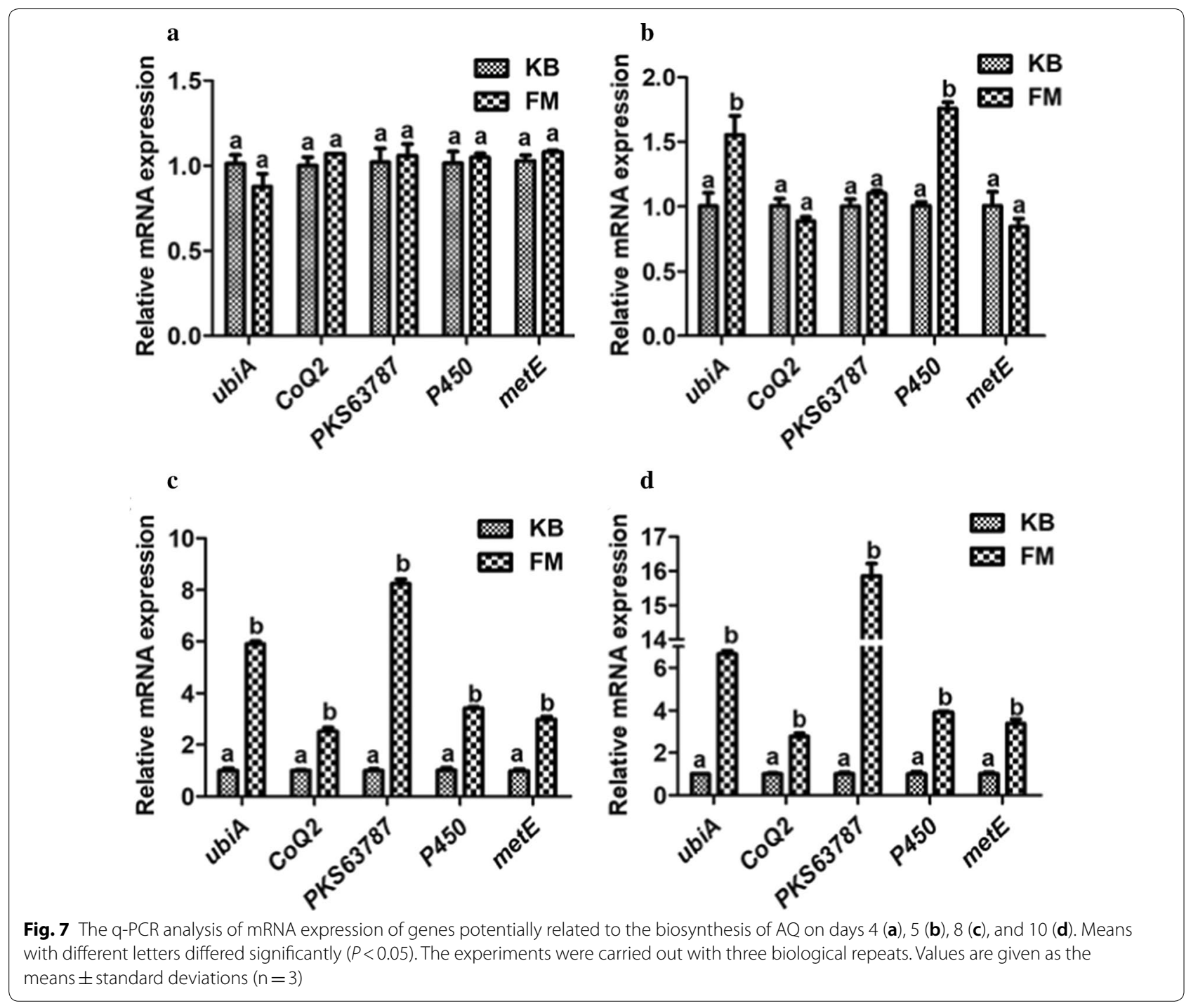

carbons, in the form of acetyl-CoA, from the fatty acid chain (Shen and Burger 2009). In addition, pyruvate is oxidized to acetyl-CoA in mitochondria during pyruvate metabolism. ACACA, E2.3.3.10, OXCT, IVD, and PAAH genes, which are associated with acetyl-CoA, were also upregulated. The formation of AQ may be closely related to the ubiquinone biosynthesis pathway (Hu et al. 2016). The initial biosynthetic pathway of ubiquinone involves the conversion of three acetyl-coA units into farnesyl diphosphate (FPP) (Ericsson et al. 1992). Therefore, acetyl-CoA may enter either the mevalonate pathway or polyketide pathway, which form the farnesyl tail precursor or ring precursor of AQ in A. camphorata S-29, respectively (Chou et al. 2017). The CoQ2 and $u b i A$ genes encode $p$-hydroxybenzoate polyprenyl transferase, which is required in the biosynthetic pathway of ubiquinone (Forsgren et al. 2004; Liu et al. 2017). P-hydroxybenzoate polyprenyl transferase, which is also referred to as the 'CoQ2 enzyme', mediates the second step in the final reaction sequence of coenzyme $\mathrm{Q}$ biosynthesis, namely, the condensation of the polyisoprenoid side chain with 4- $p$-hydroxybenzoate (Forsgren et al. 2004). The coupling of the aromatic substrate and isoprenoid chain is presumed to be the rate-limiting step in ubiquinone synthesis. Chou et al. (2019) proposed that the farnesylation of coenzyme $\mathrm{Q}_{0}$ at C-3 forms 5-demethoxy-coenzyme $\mathrm{Q}_{3}$ or coenzyme $\mathrm{Q}_{3}$, which is further reduced to form $\mathrm{AQ}$. Therefore, we hypothesize that overexpression of $\mathrm{CoQ} 2$ and $u b i A$ genes promoted the linkage of coenzyme $\mathrm{Q}_{0}$ and the polyisoprene side chain, thus increasing AQ synthesis during FM.

The iTRAQ has the advantages of high coverage, accuracy, and sensitivity. Proteomic analyses of A. camphorata S-29 using the iTRAQ technique could provide 
useful information for understanding the AQ biosynthesis pathway. Based on the numerous unique proteins identified in GO functional categories, the largest categories for the functional group were sulphur amino acid and sulphur compound biosynthetic processes (Fig. 4). In microorganisms, sulphate is used in the synthesis of organic sulphur metabolites, mostly cysteine, methionine, and $S$-adenosylmethionine. The sulphur amino acids (SAA) include methionine and cysteine (Glenda et al. 2012). Methionine is metabolized via three major metabolic pathways: transmethylation, remethylation, and transsulfuration (Vigneaud et al. 1994). Fungi utilize the methyl group of methionine for biological methylation. Methionine serves as the major methyl group donor. The expression of $S$-adenosylmethionine synthetase is significantly increased during FM of $A$. camphorata S-29 compared with control (Fig. 4). $S$-adenosylmethionine synthetase is the transferring enzyme with $S$-methyltransferase activity. In addition, upregulated metE genes could encode an enzyme (ec:2.1.1.14) with methyltransferase activity. According to the reported results, it is important to have a sufficient supply of methyl groups for AQ structure formation (Wijayasinghe et al. 2014; Hu et al. 2016). These results suggest that $S$-adenosylmethionine synthetase may play a vital role in the biosynthesis of AQ.

According to results from Yu et al. (2016), the biosynthesis of aromatic compounds in A. camphorata occurs via the polyketide pathway. PKS63787 plays a critical role in the biosynthesis of benzoquinones and benzenoids. Three metabolites, including benzenoids, benzoquinones, and AQ, are related to PKS63787. Yu et al. (2017) used phylogenetic analysis to demonstrate that PKS63787 likely encodes an orsellinic acid synthase. The biosynthetic route of AQ follows a synthetic sequence similar to that of yeast coenzyme $\mathrm{Q}_{6}$. The pathway starts with the farnesylation of orsellinic acid to form 3-farnesylorsellinic acid, which undergoes further ring modification to form 5-demethoxy-coenzyme $\mathrm{Q}_{3}$, coenzyme $\mathrm{Q}_{3}$, and finally AQ (Chou et al. 2017). Table 3 and Additional file 1: Table S2 show that PKS63787 gene expression is significantly increased in FM on day $10(P<0.001)$. After adding coenzyme $\mathrm{Q}_{0}$ on day 4 , its content gradually decreased from days $4-10$. Coenzyme $\mathrm{Q}_{0}$ was completely utilized after day 10 (Additional file 1: Figure S7). Therefore, we hypothesized that excess polyisoprene side chain induced the overexpression of the PKS63787 gene to synthesize the cyclohexanone moiety of AQ. However, further investigations are required.

CYP genes are ubiquitously distributed genes in the genomes of almost all organisms. CYP genes encode a superfamily of haeme-containing monooxygenases that metabolize a wide variety of endogenous and xenobiotic compounds (Bernhardt 2006). Many fungal species have evolved complex and diverse CYP systems that play an important role in primary and secondary metabolism, allowing these fungi to exhibit unique and superior metabolic functions (Brink et al. 1998). CYP enzyme systems of fungi also include a wide range of catalysed reactions, such as reduction, heteroatom oxygenation, epoxidation, dealkylation, aromatic hydroxylation, and carbon hydroxylation (Brink et al. 1998; Ling et al. 2017). Hsu et al. (2011) identified 10 CYP genes (ac-1 to ac-10) from A. camphorata. According to phylogenetic tree of fungal CYP amino acid sequences, ac-8 CYP was assigned to a new CYP family. Since ac-8 CYP did not exhibit close evolutionary relationships with known CYPs, the function of ac-8 CYP is unknown. In the transcriptomic and proteomic analysis, the expression of ac- 8 CYP monooxygenase increased significantly on day 10 . In addition, mRNA expression levels of $P 450$ were significantly increased in the FM compared to the KB at 5, 8 and 10 days (Fig. 6b-d). Therefore, we hypothesized that ac-8 CYP monooxygenase plays an important role in AQ synthesis.

According to several reports, coenzyme $\mathrm{Q}_{0}$ is associated with the electron transport respiratory chain (Redfeam and Burgos 1966). Several DEGs and DEPs that might be closely related to the electron transport respiratory chain were noted in this study. E1.14.13.1, $E 1.14 .13 .7$, and $A D K$ are three energy-related genes that exhibit increasing levels of expression upon the addition of coenzyme $Q_{0}$. One of the main functions of these genes is to provide energy for cells and electron transport. Farnesylation is a chemical reaction that involves covalent bonding, requiring higher energy. Overexpression of these genes may facilitate oxidative phosphorylation and accelerate electron transport through cytochrome $\mathrm{c}$ within the inner mitochondrial membrane, and finally activate ATP synthase to synthesise the energy carrier ATP. In addition to farnesylation steps, the conversion step of coenzyme $Q_{3}$ to $A Q$ involves the elimination of one double bond and the reduction of a keto group. These chemical reactions require several protons that are derived from NADH. When NADH is transformed into NAD+, this reaction releases two electrons, which can be accepted by electrophile substances, such as oxygen (Wei et al. 2020). This reaction may be beneficial for the conversion of coenzyme $\mathrm{Q}_{3}$ to $\mathrm{AQ}$.

In summary, transcriptomic and proteomic analyses showed that specific genes and enzymes associated with ubiquinone and other terpenoid-quinone biosynthesis pathways as well as various transcription factors are involved in AQ formation. Two "omics" levels of analyses identified several important candidate genes and enzymes that regulate or participate in AQ biosynthesis (Additional 
file 1: Figure S8). This study is beneficial to analyse AQ biosynthesis pathways and provide strategies for targeted increases in $\mathrm{AQ}$ production by genetic means in the future.

\section{Supplementary information}

Supplementary information accompanies this paper at https://doi. org/10.1186/s13568-020-01076-6.

Additional file 1: Table S1. Primers used for q-PCR. Table S2. Summary of DEGs in KEGG pathway annotation (10-day samples). Table S3. GO annotation of upregulated proteins (10-day samples). Table S4. Other upregulated proteins on day 10 based on proteomic analysis. Table S5. The number of genes and proteins at transcriptomic and proteomic levels (10-day samples). Table S6. The mRNA expression levels of genes on days 4 and 5. Figure S1. Venn diagram of expressed genes in A. camphorata $\mathrm{S}-29$ transcriptomes in $\mathrm{KB}$ and FM on days 4, 5, and 10, respectively. Figure S2. Functional categorization by KEGG of A. camphorata S-29 transcriptome. Figure S3. Analysis of DEGs between KB and FM by KEGG enrichment map. A. KB4 VS FM4; B. KB5 VS FM5. Figure S4. A summary of protein information of $A$. camphorata S-29. Figure S5. The GO annotation of 3987 proteins in A. camphorata S-29. Figure S6. Volcano plot of the 3987 proteins during FM compared with KB. Figure S7. The concentrations of $\mathrm{CoQ} 0$ and $\mathrm{AQ}$. The experiments were carried out with three replications. Values are given as the means \pm standard deviations $(n=3)$. Figure $\mathbf{S} 8$. Genes annotations in the AQ synthesis pathway during FM. FPP, farnesyl diphosphate; OA, orsellinic acid; FOA, 3-farnesyl-orsellinic acid; 5-DMQ3, 5-demethoxy- coenzyme Q3; CoQ3, coenzyme Q3; $A Q$, antroquinonol.

\section{Acknowledgements}

Not applicable.

\section{Authors' contributions}

$L X F, X Y$, and $A L Z$ conceived and designed research. $L X F, Z Y$, and $Y C Y$ conducted experiments. $X Z Q$ and $A L Z$ contributed new reagents or analytical tools. XZQ and SX analysed data. LXF wrote the manuscript. All authors read and approved the final manuscript.

\section{Funding}

This work was supported by the National Natural Science Foundation of China (grant number 31871757) and Shanghai Engineering Research Center of Food Microbiology (grant number 19 DZ2281100).

\section{Availability of data and materials}

The FASTQ format raw reads were deposited to the National Center for Biotechnology Information Short Read Archive (NCBI SRA) database (accession: PRJNA543624 and PRJNA622907). The mass spectrometry proteomics data have been deposited to the ProteomeXchange Consortium (http://prote omecentral.proteomexchange.org) via the iProX partner repository with the dataset identifier PXD018004.

\section{Ethics approval and consent to participate}

Not applicable.

\section{Consent for publication}

All authors agree to publish.

\section{Competing interests}

All authors declare that they no competing interests.

Received: 6 July 2020 Accepted: 28 July 2020

Published online: 03 August 2020

\section{References}

Angamuthu V, Shanmugavadivu M, Nagarajan G, Velmurugan BK (2019) Chemico-biological interactions pharmacological activities of antroquinonol-mini review. Chem Biol Interact 297:8-15

Bai B, Wu J, Sheng WT, Zhou B, Zhou LJ, Zhuang W, Yao DP, Deng QY (2015) Comparative analysis of anther transcriptome profiles of two different rice male sterile lines genotypes under cold stress. Int J Mol Sci 16(5):11398-11416

Bernhardt R (2006) Cytochromes P450 as versatile biocatalysts. J Biotechnol 124:128-145

Brink HMVD, Gorcom RFMV, Hondel CAVD, Punt PJ (1998) Cytochrome p450 enzyme systems in fungi. Fungal Genet Biol 23(1):1-17

Chang $\mathrm{WH}$, Chen MC, Cheng IH (2015) Antroquinonol lowers brain amyloid- $\beta$ levels and improves spatial learning and memory in a transgenic mouse model of Alzheimer's disease. Sci Rep 5:15067-15078

Chiang PC, Lin SC, Pan SL, Kuo CH, Tsai IL, Kuo MT, Wen WC, Chen PN, Guh JH (2010) Antroquinonol displays anticancer potential against human hepatocellular carcinoma cells: a crucial role of AMPK and mTOR pathways. Biochem Pharmacol 79:162-171

Chou KCC, Yang SH, Wu HL, Lin PY, Chang TL, Sheu F, Chen KH, Chiang BH (2017) Biosynthesis of antroquinonol and 4-acetylantroquinonol B via a polyketide pathway using orsellinic acid as a ring precursor in Antrodia cinnamomea. J Agric Food Chem 65(1):74-86

Chou KCC, Wu HL, Lin PY, Yang SH, Chang TL, Sheu F, Chen KH, Chiang BH (2019) 4-Hydroxybenzoic acid serves as an endogenous ring precursor for antroquinonol biosynthesis in Antrodia cinnamomea. Phytochemistry 161:97-106

Ericsson J, Appelkvist EL, Thelin A, Chojnacki T, Dallner G (1992) Isoprenoid biosynthesis in rat liver peroxisomes: characterization of cis-prenyltransferase and squalene synthetase. J Biol Chem 267(26):18708-18714

Forsgren M, Attersand A, Lake S, Jacob G, Climent I (2004) Isolation and functional expression of human coq2, a gene encoding a polyprenyl transferase involved in the synthesis of CoQ. Biochem J 382:519-526

Glenda CM, Ball RO, Pencharz PB (2012) Sulfur amino acid metabolism and requirements. Nutr Rev 70(3):170-175

Hsu KH, Lee YR, Lin YL, Chu FH (2011) Cytochrome P450 genes in medicinal mushroom Antrodia cinnamomea T.T. Chang et W.N. Chou (higher basidiomycetes) are strongly expressed during fruiting body formation. Int J Med Mushrooms 13(6):513-523

Hu YD, Zhang BB, Xu GR, Liao XR, Cheung PCK (2016) A mechanistic study on the biosynthetic regulation of bioactive metabolite antroquinonol from edible and medicinal mushroom Antrodia camphorata. J Funct Foods 25:70-79

Juan CV, María José ME, Eva RS, Eva B, Elortza F, Roque BM (2010) iTRAQ-based quantitative analysis of protein mixtures with large fold change and dynamic range. Proteomics 10(2):343-347

Lee TH, Lee CK, Tsou WL, Liu SY, Wen WC (2007) A new cytotoxic agent from solid-state fermented mycelium of Antrodia camphorata. Planta Med 73:1412-1415

Li B, Dewey CN (2011) RSEM: accurate transcript quantification from RNA-Seq data with or without a reference genome. BMC Bioinform 12:16

Ling YL, Ma LT, Lee YR, Shaw JF, Wang SY, Chu FH (2017) Differential gene expression network in terpenoid synthesis of Antrodia cinnamomea in mycelia and fruiting bodies. J Agric Food Chem 65:1874-1886

Liu Y, Ding XM, Xue ZL, Hu LX, Cheng Q, Chen MH, Su Y, Zhu B, Xu P (2017) Site-directed mutagenesis of $u b i A$ to promote menaquinone biosynthesis in Elizabethkingia meningoseptica. Process Biochem 58:186-192

Liu XF, Xia YJ, Zhang Y, Sang KK, Xiong ZQ, Wang GQ, Liu XX, Ai LZ (2020) Transcriptomic analyses of Antrodia camphorata to determine antroquinonol and antrodin C biosynthetic mechanisms in the in situ extractive fermentation. J Sci Food Agric. https://doi.org/10.1002/jsfa.10467

Lu MC, El-Shazly M, Wu TY, Du YC, Chang TT, Chen CF, Hsu YM, Lai KH, Chiu CP, Chang FR, Wu YC (2013) Recent research and development of Antrodia cinnamomea. Pharmacol Ther 139(2):124-156

Lu MYJ, Fan WL, Wang WF, Chen T, Tang YC, Chu FH, Chang TT, Wang SY, Li MY, Chen YH, Lin ZS, Yang KJ, Chen SM, Teng YC, Lin YL, Shaw JF, Wang TF, Li $\mathrm{WH}$ (2014) Genomic and transcriptomic analyses of the medicinal fungus Antrodia cinnamomea for its metabolite biosynthesis and sexual development. Proc Natl Acad Sci USA 111(44):E4743 
Lu ZM, Zhu Q, Li HX, Geng Y, Shi JS, Xu ZM (2017) Vanillin promotes the germination of Antrodia camphorata arthroconidia through PKA and MAPK signaling pathways. Front Microbiol 8:2048

Ma J, Chen T, Wu S, Yang C, Bai M, Shu K, Li K, Zhang G, Jin Z, He F, Hermjakob H, Zhu Y (2019) iProX: an integrated proteome resource. Nucleic Acids Res 47:D1211-D1217

MacLean D, Jones JDG, Studholme DJ (2009) Application of "next-generation" sequencing technologies to microbial genetics. Nat Rev Microbiol 7(4):287-296

Redfeam ER, Burgos J (1966) Ubiquinone (coenzyme Q) and the respiratory chain. Nature 209:711-713

Robinson MD, Mccarthy DJ, Smyth GK (2010) Edger: a Bioconductor package for differential expression analysis of digital gene expression data. Bioinformatics 26(1):139-140

ShenYQ Burger G (2009) Plasticity of a key metabolic pathway in fungi. Funct Integr Genom 9:145-151

Trapnell C, Roberts A, Goff L, Pertea G, Kim D, Kelley DR, Pimentel H, Salzberg SL, Rinn JL, Pachter L (2012) Differential gene and transcript expression analysis of RNA-seq experiments with TopHat and Cufflinks. Nat Protoc 7:562-578

Vera Alvarez R, Pongor LS, Marino-Ramirez L, Landsman D (2019) TPMCalculator: one-step software to quantify mRNA abundance of genomic features. Bioinformatics 35:1960-1962

Vigneaud D, Kilmer GW, Rachele JR, Cohn M (1994) On the mechanism of the conversion in vivo of methionine to cysteine. J Biol Chem 155:47-55

Washburn MP, Koller A, Oshiro G, Ulaszek RR, Plouffe D, Deciu C, Winzeler E, Yates JR (2003) Protein pathway and complex clustering of correlated mRNA and protein expression analyses in Saccharomyces cerevisiae. Proc Natl Acad Sci USA 100:3107-3112
Wei YY, Chou KCC, Yang SH, Chiang BH (2020) Oxygen vector accelerates farnesylation and redox reaction to promote the biosynthesis of 4-acetylantroquinonol $\mathrm{B}$ and antroquinonol during submerged fermentation of Antrodia cinnamomea. Food Bioprod Process 120:80-90

Wijayasinghe YS, Blumenthal RM, Viola RE (2014) Producing proficient methyl donors from alternative substrates of S-adenosylmethionine synthetase. Biochemistry 53:1521-1526

Wu SH, Ryvarden L, Chang TT (1997) Antrodia camphorata ("niu-chang-chih"), new combination of a medicinal fungus in Taiwan. Bot Bull Acad Sin 38:273-275

Xia YJ, Chen Y, Liu XF, Zhou X, Wang ZC, Wang GQ, Xiong ZQ, Ai LZ (2018) Enhancement of antroquinonol production during batch fermentation using $\mathrm{pH}$ control coupled with an oxygen vector. J Sci Food Agric 91:2463-2470

Xie C, Mao X, Huang J, Ding Y, Wu J, Dong S, Kong L, Gao G, Li CY, Wei L (2011) KOBAS 2.0: a web server for annotation and identification of enriched pathways and diseases. Nucleic Acids Res 39:W316-W322

Yu PW, Chang YC, Liou RF, Lee TH, Tzean SS (2016) PKS63787, a polyketide synthase gene responsible for the biosynthesis of benzenoids in the medicinal mushroom Antrodia cinnamomea. J Nat Prod 79:1485-1491

Yu PW, Cho TY, Liou RF, Tzean SS, Lee TH (2017) Identification of the orsellinic acid synthase PKS63787 for the biosynthesis of antroquinonols in Antrodia cinnamomea. Appl Microbiol Biotechnol 101(11):4701-4711

\section{Publisher's Note}

Springer Nature remains neutral with regard to jurisdictional claims in published maps and institutional affiliations.

\section{Submit your manuscript to a SpringerOpen ${ }^{\odot}$ journal and benefit from:}

- Convenient online submission

- Rigorous peer review

- Open access: articles freely available online

- High visibility within the field

- Retaining the copyright to your article

Submit your next manuscript at springeropen.com 